\title{
Prenatal detection of trisomy 21 and 18 from amniotic fluid by quantitative fluorescent polymerase chain reaction
}

Tamás Tóth, Ian Findlay, Csaba Papp, Ernõ Tóth-Pál, Tamás Marton, Bálint Nagy, Philip Quirke, Zoltán Papp

\begin{abstract}
Prenatal diagnosis of fetal trisomies is usually performed by cytogenetic analysis on amniotic fluid. This requires lengthy laboratory procedures and high costs, and is unsuitable for large scale screening of pregnant women. An alternative method, which is both rapid and inexpensive and suitable for diagnosing trisomies even from single fetal cells, is the fluorescent polymerase chain reaction using polymorphic small tandem repeats (STRs). In this paper we present the preliminary results of a larger study comparing parallel prenatal diagnoses of trisomies 21 and 18 using cytogenetics with quantitative fluorescent polymerase chain reaction using STR markers. The results obtained by the two techniques were concordant in all cases. This is the first study reporting significant numbers of prenatal diagnoses using the quantitative fluorescent polymerase chain reaction. We believe that further studies on greater numbers of samples will determine the absolute reliability of this technique. These results also provide a model for diagnosis of trisomy from single fetal cells isolated from maternal blood.

(F Med Genet 1998;35:126-129)
\end{abstract}

Keywords: Down syndrome; prenatal diagnosis; fluorescent polymerase chain reaction

Obstetrics and

Gynaecology,

Semmelweis

University Medical

School, Baross u 27,

Budapest, H-1088,

Hungary

T Tóth

C Papp

E Tóth-Pál

T Marton

B Nagy

Z Papp

Molecular Oncology, Institute of Pathology, Algernon Firth

Building, University of

Leeds, Leeds LS2 9LN,

UK

I Findlay

P Quirke

Correspondence to:

Dr Tóth.

Received 29 April 1997 Revised version accepted for publication 2 September 1997 syndrome) has the highest birth prevalence (approximately 1 affected newborn in 700-800 births ${ }^{2}$ ). Trisomy 21 results in moderate to

Table 1 Primer sequences for D21S11 and D18S51

\begin{tabular}{ll}
\hline Marker & Sequence of primers \\
\hline D21S11 (F) & 5'-TGT ATT AGT CAA TGT TCT CCA G-3' \\
D21S11 (R) & 5'-ATA TGT GAG TCA ATT CCC CAA G-3' \\
D18S51 (F) & 5'-CAA ACC CGA CTA CCA GCA AC-3' \\
D18S51 (R) & 5'-GAG CCA TGT TCA TGC CAC TG-3' \\
\hline
\end{tabular}

severe mental retardation, high risk of congenital heart disease, and other multiple malformations and is the major reason that older mothers seek prenatal diagnosis. The next most common trisomy observed in prenatal diagnosis is trisomy 18 (Edwards syndrome). Even though $\sim 90 \%$ of trisomy 18 pregnancies result in fetal death, this syndrome has an incidence of 1 affected newborn in 1000 live births and results in severe mental retardation, multiple malformations, and is generally fatal before 1 year of age.

As the frequency of chromosomal abnormalities increases with maternal age, ${ }^{3}$ trisomy screening by prenatal diagnosis (by amniocentesis or chorionic villus sampling) has become accepted by women over 35 years of age. ${ }^{4}$ However, despite the extensive care of pregnant women over 35 years of age, the birth prevalence of Down syndrome remains high, as two-thirds of Down syndrome pregnancies occur in women under 35 years of age. Therefore to reduce significantly the birth prevalence of trisomies, a wide ranging screening programme of pregnant women has been suggested.

Current methods, such as cytogenetic analysis, for detecting chromosomal abnormalities often require lengthy laboratory procedures and incur high costs as well as significant delay in providing a diagnosis. These tests are therefore not suitable for large scale screening of all pregnant women. An alternative method, which is both rapid, inexpensive, and suitable for diagnosing trisomies, is the fluorescent polymerase chain reaction using polymorphic small tandem repeats (STRs). The quantitative nature of this technique allows the amount of PCR product to be determined and thus allows peak ratios to be calculated Although the first description of this method was reported by Mansfield in $1993,{ }^{5}$ there have been only a few reports ${ }^{67}$ applying this technique clinically to trisomy detection in prenatal diagnosis.

We report our experiences using a fluorescent polymerase chain reaction based investigation of D21S11 and D18S51 tetranucleotide STR markers to determine rapidly trisomy 21 and 18 status from amniotic fluid samples.

\section{Materials and methods}

DNA PREPARATION

Cells from $1 \mathrm{ml}$ amniotic fluid were collected by centrifugation at $15000 \mathrm{rpm}$ for 10 minutes. The supernatant was discarded and $100 \mu \mathrm{l}$ of resin from ReadyAmp Genomic 
Table 2 Heterozygosity and peak areas for disomic samples. Mean is calculated from ratios obtained by dividing the larger by the smaller allele area. Of the 212 samples tested, three samples were trisomic for chromosome 18 and four samples were trisomic for chromosome 21. This table shows the disomic samples

\begin{tabular}{llllllll}
\hline \multirow{2}{*}{ Marker } & \multirow{2}{*}{ Homozygous } & Heterozygous & Total tested & Heterozygosity & Mean & $S D$ & Range \\
\hline D21S11 & 23 & 185 & 208 & 0.89 & 1.07 & 0.052 & $1.00-1.22$ \\
D18S51 & 14 & 195 & 209 & 0.93 & 1.09 & 0.064 & $1.00-1.26$ \\
\hline
\end{tabular}

Purification System (Promega, USA) was added to the pelleted cells. The subsequent extraction procedure was performed according to the manufacturer's protocol. Six microlitres from this DNA solution were used in each PCR reaction.

FLUORESCENT POLYMERASE CHAIN REACTION PCR amplification was carried out in two separate assays, one with primers for marker D21S1 $1^{8}$ and the second with primers for marker D18S51 (Genomic Data Base). Primer sequences are listed in table 1 . The D21S11 and D18S51 forward primers were labelled at the 5 ' end with 5-carboxy-fluorescein (FAM). PCRs were performed in a total volume of $50 \mu \mathrm{l}$ containing $6 \mu \mathrm{l}$ of DNA solution prepared by the Promega kit, Taq polymerase buffer (Perkin Elmer), $200 \mu \mathrm{mol} / 1 \mathrm{dNTPs}, 10 \mathrm{pmol}$ of each primer, $1.5 \mathrm{mmol} / 1 \mathrm{MgCl}_{2}$, and $1.0 \mathrm{U}$ AmpliTaq (Perkin Elmer). After denaturation at $95^{\circ} \mathrm{C}$ for five minutes, 30 cycles at $95^{\circ} \mathrm{C}$ for 45 seconds, $60^{\circ} \mathrm{C}$ for 45 seconds, and $72^{\circ} \mathrm{C}$ for 45 seconds were performed in a Perkin Elmer
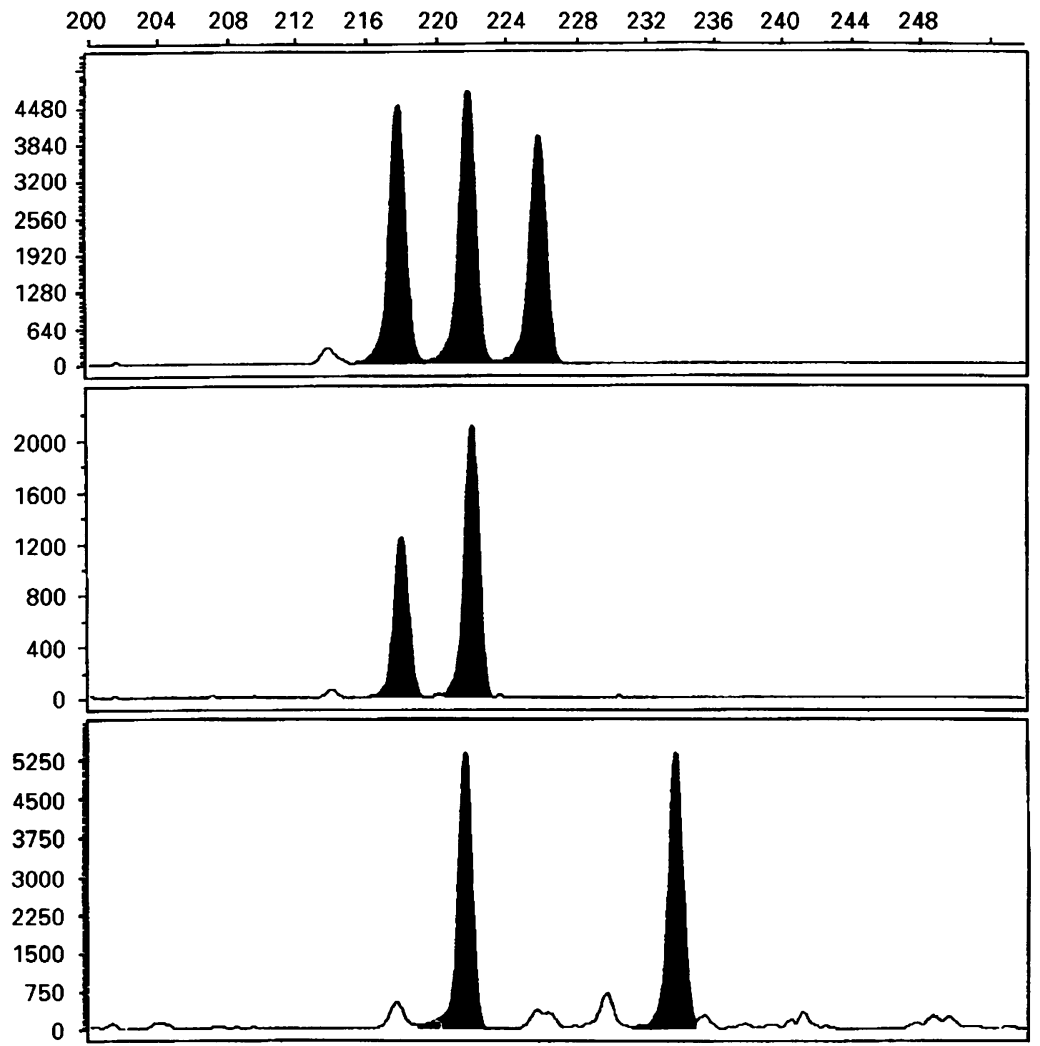

Figure 1 Electrophoretograms of D21S11 amplified from two trisomy 21 and a disomic samples. Top panel displays an electrophoretogram of a case with a triallelic pattern. Middle pamel displays a trisomy 21 fetus with diallelic pattern with a dosage ratio of $2: 1$. Bottom panel displays a normal heterozygous disomic sample with a dosage ratio of $1: 1$. The $x$ axis shows the calculated length of the PCR products. The $y$ axis shows fluorescent intensities in arbitrary units. Different fluorescent intensity scales were used for better comparison since the absolute values are different owing to the different DNA concentrations in the samples.
2400 thermal cycler. Final extension was for 10 minutes. The entire procedure took two hours.

\section{SEPARATION AND QUANTITATION OF PCR}

PRODUCTS

Two microlitres of PCR product were mixed with $24 \mu \mathrm{l}$ formamide and $1 \mu \mathrm{l}$ Genescan-500 TAMRA size standard (Applied Biosystems, USA). The mixture was denatured at $95^{\circ} \mathrm{C}$ for three minutes and placed on ice until analysis. Electrophoretic analysis was performed by using POP4 gel (Applied Biosystems, USA) and the ABI 310 Genetic Analyser (Applied Biosystems, USA). The amplification products were analysed by Genescan Analysis 2.1 software (Applied Biosystems, USA) and the relative peak areas were calculated.

\section{Results}

In a 10 week period, 212 pregnant women underwent amniocentesis to rule out fetal chromosomal abnormalities either because of maternal age or previously detected ultrasound abnormalities. Informed consent was obtained from all patients. Ten millilitres of amniotic fluid were removed; $9 \mathrm{ml}$ were used for cytogenetic analysis and $1 \mathrm{ml}$ was stored for molecular genetic analysis. Samples from each patient underwent karyotyping and trisomy 21 and 18 diagnosis using D21S11 and D18S51 STR markers, respectively.

Of the 212 samples, seven showed an abnormal karyotype according to the cytogenetic results. Trisomy 21 was detected in four samples and trisomy 18 in a further three samples. These seven pregnancies were terminated and fetal liver tissues stored for further investigations. Molecular genetic analyses were performed on the stored amniotic fluid samples by amplifying STR markers on chromosomes 21 and 18. The number of homozygous and heterozygous samples for the examined markers and relative peak areas of heterozygous samples are shown in table 2. In all cases PCR results were consistent with cytogenetic results. Two trisomy 21 (fig 1) and one trisomy 18 (fig 2) samples showed a characteristic triallelic pattern with three STR peaks of similar intensity corresponding to three different alleles, while the other two trisomy 21 (fig 1) and two trisomy 18 (fig 2) samples showed a characteristic diallelic pattern with a fluorescent intensity ratio of $2: 1$.

Although ratio ranges of normal heterozygous and diallelic trisomic samples did not overlap, diallelic trisomic samples should be investigated further using other markers on each chromosome to determine the quantitative amplification of different alleles and thus the trisomy. 
248256264272280288296304312320328336344352360368376384392
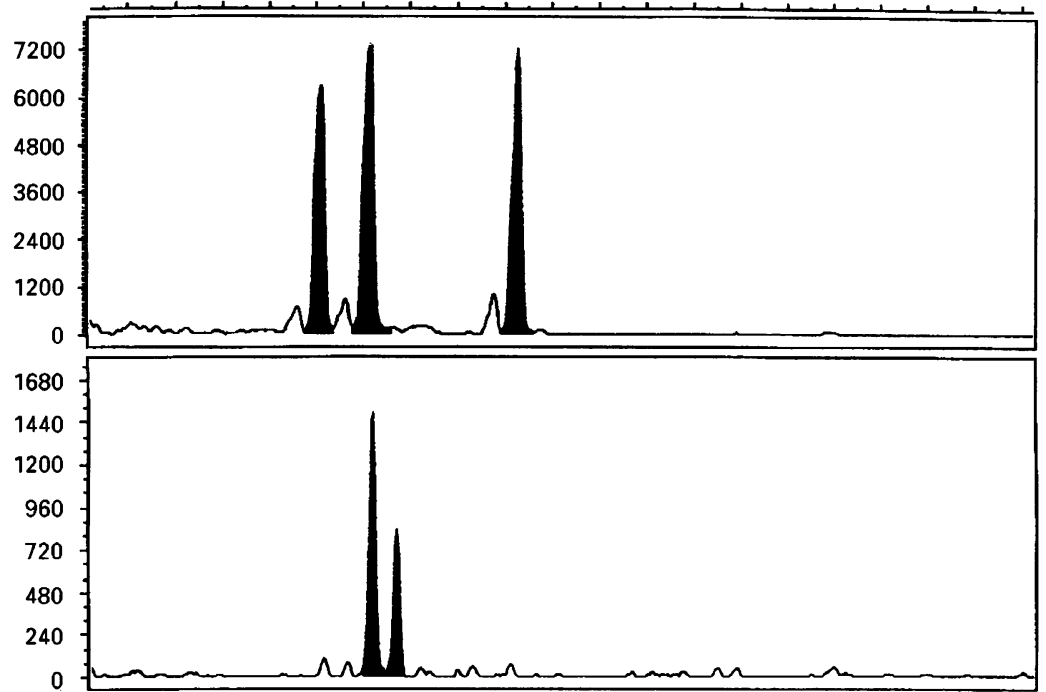

(1)

Figure 2 Electrophoretograms of D18S51 amplified from two trisomy 18 and a disomic samples. Top panel displays an electrophoretogram of a case with a triallelic pattern. Middle panel displays a trisomy 18 fetus with diallelic pattern with a dosage ratio of $2: 1$. Bottom panel shows a a normal heterozygous disomic sample with a dosage ratio of $1: 1$. Axes and scales are the same as described in legend to fig 1.

\section{Discussion}

Chromosomal abnormalities form an important group of genetic disorders. The pre- and postnatal detection of these abnormalities have been almost exclusively performed by cytogenetic analysis which requires live cells, complex conditions, and time consuming laboratory culture to obtain sufficient cells for analysis. In addition, cytogenetic detection of trisomies cannot be performed from stored tissues or from single cells.

Fluorescent in situ hybridisation (FISH) allows identification of specific chromosomes when the cells are in interphase. Although FISH can be used for detection of trisomies from amniotic fluid, stored tissues, and single cells, several technical difficulties have been reported and the evaluation of trisomic results using FISH remains to be resolved. ${ }^{9}$

Another fluorescent technique which is more reliable, accurate, and can be automated is the amplification of STRs on different chromosomes using a quantitative fluorescent polymerase chain reaction and subsequent detection of products by a DNA Sequencer. The method is based on the observation that the amount of PCR products is proportional to the quantity of initial target sequence. The first report using quantitative fluorescent $\mathrm{PCR}$ to examine trisomies dates from $1993^{5}$; however, this method has been used for prenatal diagnosis by only a few groups. ${ }^{6} 71011$

We report our initial experiences using quantitative fluorescent PCR for prenatal exclusion of trisomy 21 and 18. These preliminary experiments show that fluorescent PCR amplification of STRs can reliably be used for the detection of trisomies of both chromosomes 21 and 18. Allelic drop out, as described by Findlay et $a l,{ }^{12}$ was not observed during the PCRs (table 2, relative peak areasrange). However, to minimise the chance of misdiagnosis, the PCRs were repeated. The introduction of additional markers is also recommended and this is currently under investigation in our laboratory. STR markers on other chromosomes could also be used in a similar manner allowing detection of other chromosomal abnormalities by molecular genetic methods rather than cytogenetic analysis.

We believe that using two highly polymorphic STR markers on each chromosome would allow diagnostic accuracy to be increased in most of the cases investigated. In noninformative cases further polymorphic marker(s) could be amplified. We are currently using a multiplex PCR assay with several STR markers for chromosomes 21,18 , and 13 which will allow this technique to become even more valuable in the prenatal detection of trisomies. Quantitative fluorescent PCR was used in parallel with cytogenetic analysis for prenatal detection of trisomies as a "back up" in case of culture or cytogenetic analysis failure.

This technique allows detection of trisomies within four hours in urgent cases, allowing amniocentesis and possible termination to be undertaken on the same day. This is especially important when the pregnancy is relatively advanced as delay may lead to gestation exceeding the legal limit for termination. Amniocentesis takes about 10 minutes and DNA preparation from amniotic fluid takes only 50 minutes using the DNA purification system (Promega, USA) instead of a long phenol/chloroform extraction procedure. The length of PCR is two hours and the detection of one sample by the ABI 310 DNA sequencer takes 30 minutes. A time limit can be very important, for example in cases of preimplantation diagnosis.

As fluorescent PCR can also be performed from single cells, ${ }^{13}{ }^{14}$ this technique appears to be suitable for prenatal diagnosis of trisomies from isolated fetal cells from maternal blood ${ }^{15}$ or in preimplantation diagnosis from blastomeres obtained by embryo biopsy. Further investigations, which are in progress, will determine the reliability of this technique in these fields.

We would like to thank the Medical Research Council for grant (MRC G9530631) and the Special Trustees of the United Leeds Teaching Hospitals for financial support.

1 Hook EB. Chromosome abnormalities. In: Brock D, Rodeck C, Ferguson-Smith M, eds. Prenatal diagnosis and screening. C, Ferguson-Smith M, eds. Prenatal diagn

2 Hook EB. Rates of chromosome abnormalities at different maternal ages. Obstet Gynecol 1981;58:282-5.

3 Hook EB. Down syndrome rates and relaxed selection at older maternal ages. Am f Hum Genet 1983;35:1307-13.

4 Ferguson-Smith MA, Yates JRW. Maternal age specific rates for chromosome aberrations and factors influencing them: report of a collaborative European study on 52965 amniocenteses. Prenat Diagn 1984;4:5-44.

5 Mansfield ES. Diagnosis of Down syndrome and other aneuploidies using quantitative polymerase chain reaction 
and small tandem repeat polymorphisms. Hum Mol Genet 1993;2:43-50.

6 Pertl B, Yau SC, Sherlock J, Davies AF, Mathew CG, Adinolfi M. Rapid molecular method for prenatal detection Adinolf M. Rapid molecular method for prenatal

7 Pertl B, Weitgasser U, Kopp S, Kroisel PM, Sherlock J, Adinolfi $M$. Rapid detection of trisomies 21 and 18 and sexing by quantitative fluorescent multiplex PCR. Hum Genet 1996;98:55-9.

8 Sharma V, Litt $M$. Tetranucleotide repeat polymorphism at the D21S11 locus. Hum Mol Genet 1992;1:67.

9 Kuo WL, Tenjin H, Segraves R, Pinkel D, Golbus MS, Gray J. Detection of aneuploidy involving chromosomes 13, 18, or 21 , by fluorescence in situ hybridization (FISH) to interphase and metaphase amniocytes. Am $\mathcal{f}$ Hum Genet 1991;49:112-19.

10 Adinolfi M, Sherlock J, Pertl B. Rapid detection of selected aneuploidies by quantitative fluorescent PCR. Bioessays 1995;17:661-4.
11 Eggeling F, Freytag M, Fahsold R, Horsthemke B, Claussen U. Rapid detection of trisomy 21 by quantitative PCR. Hum Genet 1993;91:567-70.

12 Findlay I, Ray P, Quirke P, Rutherford AJ, Lilford R. Allelic drop-out and preferential amplification in single cells and human blastomeres: implications for preimplantation diagnosis of sex and cystic fibrosis. Hum Reprod 1995;10:1609 18.

13 Findlay I, Quirke P. Fluorescent polymerase chain reaction. Part I. A new method allowing genetic diagnosis and DNA fingerprinting of single cells. Hum Reprod Update 1996;2: 137-52.

14 Findlay I, Urquhart A, Quirke P, Sullivan KM, Rutherford AJ, Lilford R. Simultaneous DNA fingerprinting, diagnosis of sex and single-gene defect status from a single cell. Hum Reprod 1995;10:1005-13.

15 Cheung MC, Goldberg JD, Kan YW. Prenatal diagnosis of sickle cell anaemia and thalassaemia by analysis of fetal cells in maternal blood. Nat Genet 1996;14:264-8. 